\title{
Necrotising fasciitis: a rare complication of acute appendicitis
}

\author{
Thomas WY Chin *, MB, ChB, Koel WS Ko, MB, BS, KH Tsang, MB, BS, FHKAM (Radiology) \\ Department of Radiology and Imaging, Queen Elizabeth Hospital, Yaumatei, Hong Kong \\ *Corresponding author: twychin@gmail.com
}

Hong Kong Med J 2018;24:535-7

DOI: 10.12809/hkmj166180

\section{Introduction}

Acute appendicitis is a common surgical emergency that can cause severe complications if diagnosis and management are delayed. ${ }^{1}$ Necrotising fasciitis (NF) is a necrotic infection involving deeper layers of the skin and subcutaneous tissue that spreads rapidly along the fascia, progressing to systemic sepsis. It is most commonly induced by injury and is an extremely rare complication of acute appendicitis. In this article, we present a case of perforated appendicitis complicated by right thigh and scrotal NF. Since the condition runs a fulminant clinical course, prompt diagnosis and early surgical intervention are crucial to reduce mortality.

\section{Case report}

A 60-year-old man with good past health presented to the Accident and Emergency Department of Queen Elizabeth Hospital, Hong Kong, in December 2015 with a 6-day history of fever and lower abdominal pain.

His vital signs on presentation were as follows: systolic blood pressure $97 \mathrm{~mm} \mathrm{Hg}$; diastolic blood pressure $59 \mathrm{~mm} \mathrm{Hg}$; pulse 90 beats per minute; and body temperature $38.3^{\circ} \mathrm{C}$. Physical examination revealed lower abdominal and right thigh tenderness without subcutaneous emphysema. Laboratory data showed leucocytosis $\left(15.5 \times 10^{9} / \mathrm{L}\right)$ with neutrophilia (neutrophils $88.7 \%$ ) and evidence of acute kidney injury (estimated glomerular filtration rate $38 \mathrm{~mL} /$ $\mathrm{min} / 1.73 \mathrm{~m}^{2}$, serum creatinine $171 \mu \mathrm{mol} / \mathrm{L}$ ).

Abdominal, pelvic, and right hip radiographs were unremarkable. Urgent abdominal and pelvic computed tomographic (CT) scan revealed a dilated retrocaecal appendix with heterogeneous wall enhancement, compatible with acute gangrenous appendicitis. There was rupture at the appendiceal tip, contiguous with a $2.8-\times 4.5-\times 8.0-\mathrm{cm}$ gascontaining abscess posterolateral to the ascending colon and caecum (Fig 1). Increased periappendiceal and pericaecal stranding and free gas were noted. Multiple smaller pelvic abscesses were also observed. An incidental finding of abnormal swelling of the right upper thigh was noted in the limited coverage of the thigh in the original CT

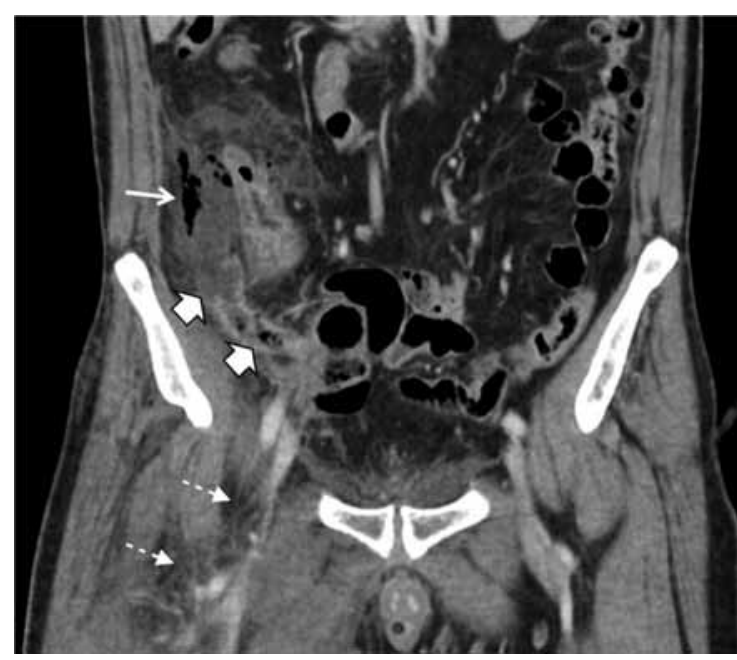

FIG I. Coronal computed tomography image: dilated retrocaecal appendix with rupture at the tip (thick arrows). The appendiceal tip connected with a gas-containing abscess (thin arrow) posterolateral to the ascending colon.Abnormal fat stranding along the femoral neurovascular bundles (dashed arrows) is shown

scan. Another CT scan including the whole right thigh was performed by the on-site radiologist. Abnormal high-density fluid and fat stranding were noted in the subcutaneous layer and intermuscular fascial planes in the anterior, adductor, and posterior compartments of the right thigh (Fig 2). The thigh muscles were swollen without discrete abscess formation. No gas density was detected along the fasciae. The initial overall imaging diagnosis was ruptured acute appendicitis with peri-appendiceal abscess, complicated by right thigh NF.

Subsequent urgent laparotomy revealed a ruptured inflamed retrocaecal appendix and an 8-cm retrocolic abscess. Appendectomy and abscess drainage were performed. These were followed by exploration of the right thigh that revealed oedematous and necrotic subcutaneous and deep soft tissues mainly involving the adductor compartment. Multiple intramuscular abscesses 


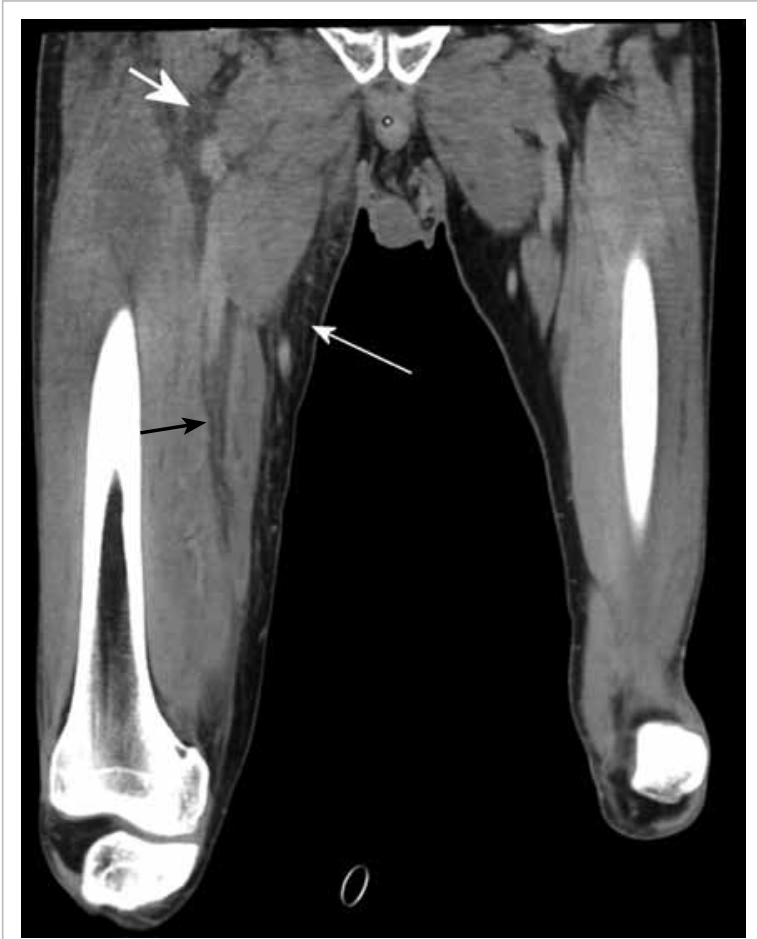

FIG 2. Coronal computed tomographic image: generalised right thigh swelling. Abnormal high-density fluid and fat stranding in the subcutaneous layer (white thin arrow), intermuscular fascial planes (black arrow) and along the femoral neurovascular bundles (white thick arrow) are shown were seen with turbid fluid tracing along the femoral neurovascular bundles proximally to the pelvic region. Overall features were compatible with NF. Right above-knee amputation with excisional debridement was performed in view of the patient's poor general condition and extensive involvement.

Culture of the debrided muscle and fascial tissue yielded Escherichia coli, Bacteroides fragilis, and Streptococcus milleri. A combination of antibiotics was given, including amikacin, ampicillin, levofloxacin, linezolid, meropenem, and penicillin G. However, septic shock persisted with development of multi-organ dysfunction despite multiple inotropes. Another CT scan of the abdomen and pelvis was performed on postoperative day 1 , revealing a grossly swollen scrotum with hydrocoele (not shown). No intrascrotal gas density was detected. In view of the patient's clinical deterioration, urgent scrotal exploration was performed and revealed extensive scrotal skin necrosis with copious serous fluid draining from the perineum.

After the second surgery, the patient had persistent multi-organ failure with clinical deterioration despite supportive management. He developed acute respiratory distress syndrome with respiratory failure and disseminated intravascular coagulation with severe anaemia, further complicated by acute coronary syndrome. The patient succumbed on the fourth day of hospital admission.

\section{Discussion}

The incidence of NF has risen recently owing to an increased prevalence of patients who are immunocompromised secondary to diabetes mellitus or treatment for malignancy or human immunodeficiency virus infection. ${ }^{1}$ The mortality rate for NF is high, despite significant advances in antibiotic treatment, owing to its rapid progression to septic shock and multi-organ failure. ${ }^{1}$

Common causes of NF include minor trauma, skin infection, intravenous drug use, and surgical complication. ${ }^{1}$ The abdomen, groin, and extremities are the regions most commonly affected by NF.1-5 Necrotising fasciitis is commonly polymicrobial, caused by virulent toxin-producing bacteria such as Group A haemolytic streptococci and Staphylococcus aureus. Other causative bacteria include Bacteroides, Clostridium, Enterobacteriaceae, Peptostreptococcus, Proteus, Pseudomonas, and Klebsiella. ${ }^{1}$

The diagnosis of NF is challenging due to its rarity and non-specific presentation. Usually the early manifestations are local inflammatory signs and symptoms such as swelling, erythema, and tenderness, occasionally with fever. However, NF should be suspected when the severity of pain or clinical status are disproportional to local findings. ${ }^{5}$ Laboratory studies show leucocytosis with predominant neutrophilia, ${ }^{1}$ but is unfortunately non-specific. ${ }^{5}$ Presence of soft tissue gas in the absence of penetrating trauma suggests a diagnosis of $\mathrm{NF}^{2}$ although the absence of soft tissue gas does not exclude the diagnosis, as evidenced by our case.

Plain radiographs are usually unhelpful for initial diagnosis as soft tissue gas is seldom detected until late in the disease process. ${ }^{1}$ Magnetic resonance imaging is superior in delineating soft tissue pathology but is a suboptimal modality for critically ill patients. ${ }^{2}$ Computed tomography is the preferred imaging modality, with reported findings including asymmetric fascial thickening with fat stranding and subcutaneous gas tracking along fascial planes. ${ }^{2}$ It also delineates the extent of tissue involvement well and is useful for monitoring treatment response.

Necrotising fasciitis secondary to perforated appendicitis is rarely reported. We have found only 18 case reports of NF caused by acute appendicitis published in the English literature. ${ }^{1-5}$ The affected regions included the abdominal wall (most commonly involved), perineum, and thigh. According to Taif and Alrawi, ${ }^{5}$ there have been only three reported cases of appendicitis complicated by NF predominantly involving the lower limb. All cases involved the right thigh, likely from more direct infective spread along 
the right femoral neurovascular bundles than the left.

Our case represents a rare but life-threatening consequence of a common disease despite the absence of risk factors. Early clinical changes of NF can be subtle. Early recognition, broad-spectrum antibiotic treatment, and aggressive surgical debridement are the cornerstones of management for this potentially lethal disease. ${ }^{1}$ A high index of suspicion is needed in diagnosis such that emergent surgical intervention can be initiated to improve clinical outcome.

\section{Author contributions}

All authors contributed to the design, acquisition and interpretation of data, drafting of the article, and critical revision for important intellectual content.

\section{Acknowledgement}

We would like to thank the staff radiographers of the Department of Radiology and Imaging, Queen Elizabeth Hospital for their assistance in acquisition of the images.

\section{Declaration}

All authors have disclosed no conflicts of interest. All authors had full access to the data, contributed to the study, approved the final version for publication, and take responsibility for its accuracy and integrity.

\section{References}

1. Chen CW, Hsiao CW, Wu CC, Jao SW, Lee TY, Kang JC. Necrotizing fasciitis due to acute perforated appendicitis: case report. J Emerg Med 2010;39:178-80.

2. Hung YC, Yang FS. Necrotizing fasciitis-a rare but severe complication of perforated appendicitis. Radiol Infect Dis 2015;2:81-3.

3. Hua J, Yao L, He ZG, Xu B, Song ZS. Necrotizing fasciitis caused by perforated appendicitis: a case report. Int J Clin Exp Pathol 2015;8:3334-8.

4. Wanis M, Nafie S, Mellon JK. A case of Fournier's gangrene in a young immunocompetent male patient resulting from a delayed diagnosis of appendicitis. J Surg Case Rep 2016;2016:rjw058.

5. Taif S, Alrawi A. Missed acute appendicitis presenting as necrotising fasciitis of the thigh. BMJ Case Rep 2014;2014:bcr2014204247.

\section{Answers to CME Programme Hong Kong Medical Journal August 2018 issue}

Hong Kong Med J 2018;24:408-15

I. Guidance on the management of familial hypercholesterolaemia in Hong Kong: an expert panel consensus viewpoint
A
1. True
2. False
3. True
4. False
5. False
B
1. True
2. False
3. True
4. False
5. True

Hong Kong Med J 2018;24:416-22

II. Joint recommendations on management of anaemia in patients with gastrointestinal bleeding in Hong Kong
A
1. False
2. False
3. False
4. True
5. True
B
1. False
2. True
3. True
4. True
5. True 